\title{
Environmental Decision Support Systems: Exactly What Are They?

\author{
Workshop Report
}

\author{
David A. Swayne ${ }^{1}$, R. Denzer ${ }^{2,}$ L. Lilburne ${ }^{3}$, M. Purvis ${ }^{4}$, Nigel W.T.Quinn ${ }^{5}$, A. \\ Storey ${ }^{6}$ \\ ${ }^{I}$ Computing and Information Science, University of Guelph, Canada \\ 'Saarland State University for Applied Science, Germany \\ ${ }^{3}$ Landcare, New Zealand \\ ${ }^{4}$ University of Otago, New Zealand \\ ${ }^{5}$ Lawrence Berkeley National Laboratory, USA \\ ${ }^{6}$ ES Aquatic Inc., Guelph, Canada
}

Key words: Environmental decision support systeme

Abstract: During the 1999 International Symposium on Environmental Software Systems (ISESS), held at the University of Otago, Dunedin, New Zealand, a two day workshop was conducted to examine the nature and characteristics of environmental decision support systems (EDSS). A reasonable informal definition of the composition, effectiveness, characterization and future of EDSS was captured from a fruitful discussion. Four of the participants contributed in writing to the discussion.

\section{INTRODUCTION AND MOTIVATION}

The call for participation in the ISESS workshop Environmental Decision Support Systems: Exactly what are they? Claims: "Decision support systems are the subject of a large number of major projects world-wide. Many of the papers at ISESS 1999 speak of them. Two central questions in their definition are: 'What do they contain?' and 'What distinguishes them from any other information system?' Is it a strong AI component? Is it what they deliver (advice on a decision)? Are they necessarily composed of a modelling or a monitoring subsystem? Are they robust and general purpose or do they require a programming staff to answer unanticipated questions?" 
ISESS 1999 delegates were invited to "explore the meaning of DSS from an environmental software perspective, and (if we agree on what they are) to comment on the sort of software suite(s) that would adequately serve as EDSS development tools."

A definition of EDSS that follows seems to capture the essential difference between an Environmental Information System (EIS) and a system that supports decision-making. It was a surprisingly difficult discussion: "An Environmental Decision Support System (EDSS) is an information system containing at least one component whose purpose is to support human decision making about an environmental issue."

\section{WHAT IS AN EDSS?}

An Environmental Decision Support System is a DSS that is within a broadly defined "environmental" domain. An EDSS that crosses over into some other domain or sector is no longer an EDSS but a more general DSS. A DSS has a clearly defined end user with a problem - as such it solves a predetermined problem - hopefully in a flexible manner.

The EDSS contains only those functions/operations that are part of the process of supporting the decisions concerning the given problem. It implements expertise to interpret and assess, and finally provide a synopsis or summary of the relevant information in a format that is intelligible to the decision maker. Ideally, it uses appropriate and comfortable language/jargon, and provides any necessary visualization and documentation.

There is no obligatory tool or system within an EDSS. It is not necessary to have an AI component. It would be usual to have a database component either within the EDSS or an associated EIS. It may (or may not) include simulation models.

The key characterising attribute of EDSS is that it provides options, or scenarios, for the decision maker. It should not make the decision for the user. Even with optimisation - the other options should be available for consideration by the decision maker. Each option will have biases, assumptions and even value judgements. They must be transparent. The EDSS should mirror the user's decision making process - then it is more likely to be successful. The EDSS may solve a set of complementary decisions and may support more than one class of decision maker but the more functionality/users it supports, the closer it gets to being a generic tool (i.e. an EIS). One of the participants described two scenarios: a EDSS being the interpretive tool that interfaces with an EIS to provide information for a decision maker, or a EDSS being the whole package, including the EIS. 
Other issues surfaced in the discussions. The author (of this section) disagrees that EDSS must have a geo component. This author dislikes like the term for the high expectations it promotes, to the point of changing terminology from DSS to something general like 'tool'. The nature of environmental informatics is likely to be the most important reason for difficulties in EDSS development and expectations.

\section{EDSS - SOFTWARE ENGINEERING ISSUES - ANOTHER PERSPECTIVE}

The fundamental ideas behind decision support systems emerged in the 1970s and became encoded in standard business school curricula by the early 1980s. The context for these systems was the office environment of business managers, and this provided the background and the image of the ways decision support systems were supposed to operate. The systems focused on management information; selecting the relevant information with the right amount of detail. It was presented visually to the manager so that he or she could make an informed decision. In this respect decision support systems primarily automated procedures that could, in principle, be performed manually. DSS have traditionally had two main tasks: selecting information from available data sets and making it available for inspection by the analyst; and building simple analytical models and applying them to the data to examine the consequences of the model. Although over time, more complex modelling has been incorporated into decision support systems, the general impression of DSS today evokes the image of the first of these tasks: a system that basically provides a business manager with ready access to information so that a timely decision could be made.

The modelling task in EDSS plays perhaps a larger role than in traditional DSS, but it nevertheless can be argued that environmental decision support systems are charged with essentially the same tasks as traditional DSS. There are some differences of scale associated with EDSS (when compared with traditional DSS) that fundamentally alter the nature of how they are used and how they need to be constructed.

We can identify three aspects of EDSS that make them significantly different from traditional DSS: the scale of the data sets; the complexity of the data sets; and the association with physical reality of the data sets.

Scale. The scope and scale of the tasks in the environmental arena are so large and complex, that we are now talking about activities that could not, even in principle, be carried out manually. For example environmental decision support systems must deal with multiple, large, and heterogeneous data collections that have been collected at different times, according to differing scales of granularity, and for different purposes. The first task, 
then, that of selecting appropriate information from these multiple data sets, becomes a fundamental, almost intractable problem. In this case it is not only a matter of selecting information, it is a matter of discovering knowledge from within these data sets.

Complexity. In the past decades we have not only greatly increased our capacity to collect spatially distributed data automatically, we have also significantly increased our recognition of the complex and interconnected manner in which all the spatially distributed elements interact. We now have an expanded understanding of how many individual entities interact with each other on a physical, chemical, and biological basis. We also have a greater appreciation of how large-scale interactions can lead to chaotic fluctuations that could not, even in princple, be predicted from initial states. Consequently, we are now faced with the task of managing the natural environment by taking into account all these spatially distributed, interacting elements and predict the consequences of various management scenarios.

Association with physical reality. A key aspect of environmental information is its essential connection to the natural world. Environmental data is, for the most part, not man-made, such as the data associated with a financial transaction or a property boundary. Instead, it is information that has been extracted from a complex physical process that we can never understand perfectly. Our understanding is based on mental models of a physical process. The models are usually not explicitly stored along with the data. When the data is put to use, the model is unavailable. When there are multiple sources involved, one is often dealing with separate data sets, each of which is associated with a physical model that may not be easily integrated into a common framework. For example there might be one data set that identifies 'contaminating' substances in rivers that are associated with increased bacteria growth. Another data set might associate 'contamination' with heavy metals and have an altogether different set of substances identified.

The following three characteristics of environmental data and the associated problems of analysis raise specific issues in connection with the software engineering of EDSS: the importance of user involvement in the software engineering process; the importance of metainformation; and the importance of an open, distributed architecture.

Because of the multiple sources of data that almost invariably characterize EDSS, there is a multidisciplinary nature to these systems. It will often be the case that the intended end-user will not be familiar with some aspects of the manner in which some of the data has been collected, and that person will invariably be suspicious of the results that are ultimately derived from the system. The EDSS will have to "bend over backwards" to allay the fears and doubts of users by supplying them with all the supporting 
evidence that is used in connection with a model evaluation. It is therefore essential to have the end-user involved from the earliest stages of software development so that the user's perspective can be incorporated into the design of the system and the implementation of the user interface. The environmental decision support system should be customised to meet the needs and demands of the particular decision makers who need to access the data. Modelling assumptions must be explicit. Uncertainties must be specified and quantified. An EDSS system should be constructed with the intention of facilitating exploration on the part of the user, instead of actually suggesting (or, worse, making) a decision. For example, a EDSS should allow a user to examine the boundaries of confidence with respect to a model by facilitating sensitivity analyses.

The heterogeneous nature of the data sources requires that significant efforts be made to secure, integrate, and display all available metadata associated with the data sets. Beyond information about the ownership and creation dates of a data set, what is further needed is information about the accuracy, calibration, and meaning of individual fields within the data. Software tools need to be present that facilitate the electronic input of metadata from hardcopy sources. There also need to be tools that assist in the integration of metadata from multiple data sources.

Metainformation about models is also significant. Every modelling technique that is employed will have assumptions and qualifications that should be recorded and included as metainformation that can ultimately be examined by the users of the EDSS. The complexity and physical basis of environmental data means that it is necessary to employ a collection of effective modelling techniques. A basic modelling technique that has so far provided considerable assistance in the handling of spatial data complexity is the conceptually simple, but powerful, technique of overlaying visual maps of data and displaying them to the analyst. Spatial overlay has been so widely useful that it has sometimes been assumed to be intrinsic to any environmental decision support system. It is our view, though, that rather than characterizing EDSS in terms of techniques to be applied - and the use of maps is, admittedly, a very useful technique - it is preferable to analyze in terms of the nature of the problem space. Thus, we would argue that one basic characteristic of EDSS is, not the use of maps, but the necessity of having to deal with the complexity of spatially distributed data.

In addition to the mapping and statistical modeling techniques that have been traditionally employed, there is a growing assortment of novel approaches that are becoming available as realistic modelling options. Some of these techniques entail the use of neural and evolutionary computing that are being developed in various research laboratories. The techniques are necessary components in the task of data-trawling through large data sets in 
order to carry out knowledge discovery. In order to incorporate such new computing modules, an open, distributed computing architecture is necessary for environmental decision support systems of the future. Earlier approaches towards developing large, monolithic geographical information systems are now being replaced by open, modular systems that allow the incorporation of new computing modules by means of distributed object computing, such as CORBA, DCOM, and agent-based systems.

These open architectures also facilitate the load-balancing across a distributed collection of processors that is sometimes necessary when computationally expensive techniques, such as genetic algorithm approaches, are employed. What once had to be left to the domain of the supercomputer is now a realistic computing option in a networked environment of workstations. A further advantage of a distributed architecture is the fact that is facilitates mechanisms for access to various data sets that may be stored on different platforms and according to differi ng formats.

One important and useful side-effect of distributed approaches to data ownership, modelling and data mining is the ownership, residence and particularly the physical proximity of a technique to its human expertise resource. In the same way as load-balancing in air cargo informatics is outsourced by airlines to centres of expertise, future EDSS might use the resident capability of a spatial informatics research laboratory to explore issues hidden in large data sets, at the laboratory with the expertise. This is a direct analogy to sending water samples to an accredited chemistry laboratory.

We can say, then, the environmental decision support systems of the future will be successful according to the degree to which they can be developed in harmony with the needs of the their end-user decision-makers. Of course, the development of an attractive user interface is always important for the early acceptance of a system. The ultimate success of a system may be just as significantly affected by the degree to which metainformation is used to facilitate the integration of multiple data sources and whether the system offers an open, distributed architecture enabling it to evolve to meet the changing conditions of the computational environment.

\section{DEVELOPMENT PROCESS ISSUES}

Since the current EDSS are often prototypical in nature, including multiple models which may or may not be fully verified, it is important for the developer to maintain good software development practices.

Fast prototyping of the interface allows the user to see how they will interact with the system, and provides the developer with much needed 
feedback in a timely manner. Prototyping of the underlying models allows the model designers to verify and test model interactions (if multiple models are being used) Loose-coupling of the application interface and underlying models is highly desirable. The underlying models are likely to change during the development cycle. Maintaining a loosely coupled, componentbased approach will allow substitution, modification or enhancement of the application. It is also likely that new or enhanced functionality will be requested after the initial deployment of the system. This may occur due to the discovery of variants of the initial problem or application of the system to new geographic regions. The system should allow relatively easy integration of new models as well as changes to the interface. There are many third party interface and non-interface components available, and the number is continuously growing. Openness (at least in binding new components into existing systems) remains essential to successful growth and development in EDSS.

Typical end users are policy makers and may have little or no knowledge of software configuration. It is important that the system be robust, easy to install, use sensible default values and have good documentation. Because of the complexity of an EDSS, this end user support is critical. The user needs to have either direct access to an expert or extensive documentation on the system. The documentation should cover both the technical aspects of using the system and the theoretical background so the user understands the complexities of the results, particularly the sources of error. Contextsensitive help, video clips, demonstration clips, realistic sample data, "wizards", and prior case studies all have a significant supporting role in demonstrating the techniques. Tracing or "logging" of the current decision support process steps within the system can also be an important issue. Sometimes, the pathway to a result is as important as the result itself. This trace of the decision process may be particularly important for publication of the results.

\section{EDSS AND "RATIONAL AGENT" ARCHITECTURE}

The agent approach to software has a strong following in the artificial intelligence community (Russell 1995). An approach to developing an understanding of EDSS would be to place, initially, the human decisionmaker in the role of agent. The agent is working on an environment (i.e. that over which he / she assumes responsibility for decisions taken). The agent evaluates inputs or percepts and makes decisions (a decision-making agent) which affect the environment. There is a goal (an "improved" environment), a performance measure (perhaps minimization of cost or lost employment) 
and a utility measure - a step-wise evaluation of progress towards some mission or goal $(10 \%$ reduction in $\mathrm{X})$.

As the agent's environment becomes more demanding, complex or perhaps the percepts become insufficient for rational decision-making, a computer program agent assumes increasing workload in evaluation of percepts and percept sequences. There is no inherent reason, other than unwillingness (and possibly liability), not to have tested decision systems assume responsibility previously that of the human decision-maker.There are now numerous medical decision support systems that play major roles in offloading routine diagnoses from human agents. The inherent rational behaviour of these systems exceeds that of harried medical personnel, provided limitations on diagnoses are recognized and respected. As decision support, they allow this human resource to be used more rationally on the difficult cases. Aircraft collision avoidance systems perform mission critical decisions, in time-frames not acceptable for human response. If they "get it wrong" the consequences are not palatable, but humans would not be able to compete, regardless. In environmental assessment, it is possibly not a "giant leap" from filling in a form to evaluating the form inputs automatically. Whenever complexity exceeds the human capacity to resolve the information ("percept overload"), automatic decision support is a prime candidate.

The possible scenarios are endless. Decision support can play the role of system model, of background information provider (the percept handler / filter), and can even make some more routine decisions in place of the human agent. Whether automation is horizontal (filtering and simplifying across the percept range) or vertical (assuming complete responsibility for some decisions), it is clear that EDSS systems are developing new complexities, and assuming new responsibilities

DSS agents depending on pure formal logic are not so successful (in the short term) as those specifically designed with decision-theoretic concepts in mind. Agents that make decisions are well-described in, for example (Russell 1995).

Finally, from the overall environmental perspective, AI research has resulted in formal planning systems, and systems that use well-founded principles of decision theory (Russell 1995). Environmental scientists must be cognizant of the fact that those who sit opposite them in project development (be it airports or sporting venues) are using planning tools whose utility and performance measures may not adequately protect the environment. Here, no knowledge is indeed a dangerous thing. 


\section{FUTURE DIRECTIONS FOR ENVIRONMENTAL DECISION SUPPORT SYSTEMS}

The concept of an EDSS is still a problematic to many. The sequential logic that is expected of any scientific decision making process is often difficult to discern. Putting aside semantics concerns, the central issue is not whether these tools are capable of helping the decision maker, but whether these tools continue to be used by the decision maker.

Not every environmental information system or environmental decision support system has proved useful. The majority of them are failures. There is often a lack of understanding or sensitivity to the questions being asked, inadequate familiarity with the person or persons doing the asking and the lack of flexibility built in to the software to adjust to changing conditions. These failures are often bred into environmental decision support system development process at the very beginning. Despite these failures the same mistakes continue to be made. Hence the challenge during the next decades will be less about technology and more about making the decision support systems as nimble and flexible in their solution processing as humans are in theirs. Herein lies the challenge - for each decision-maker within the decision making process must see rational behaviour in the system being deployed.

Some inroads have been made into emulation of the decision making process using expert systems and neural networks. Implementation of these systems for environmental decision making has been beset by the major problems of finishing the task and convincing decision makers to trust these systems. Many environmental planning decisions are still made in the political arena and environmental software scientists have always been reluctant to pollute the purity of their creations with the muddier waters of political and economic expediency. The best that can be said for many of these tools is that they provided an education for those involved in building them and hence made them a more useful resource to those making environmental decisions.

The future will require much more multidisciplinary involvement in software development than has occurred in the past. Although lip service has been made to the importance of involving those from other disciplines in the development of software, this involvement typically occurs in the final stages after most of the important software architecture decisions have been made. Even when outside disciplines are involved, the involved are most often those individuals that are most accessible and easiest to work with rather than those involved in decision making (and often the hardest from whom to gain knowledge). Hence the challenge remains to build robust, 
flexible, appropriate tools that will evolve over time as the conditions upon which environmental decisions are based evolves.

\section{ACKNOWLEDGEMENTS AND COMMENTS}

Participants in the workshop were: R. Denzer, D. Lam, L. Lilburne, M. Purvis, N. Quinn, H. Sirvio, G. Schimak, A. Storey, S. Veitch, D. Swayne (chair), W. Walley. Those named as report co- authors made significant contributions to this discussion for which the session chair is grateful. A web-site and a listserv on EDSS are being developed.

\section{REFERENCES}

Russell (1995), Artificial Intelligence: A Modern Approach, Stuart Russell and Peter Norvig, Prentice Hall 1995.

Densham (1991), Densham, P. J. (1991). Spatial decision support systems. In Geographical information systems: principles and applications, D. J. Maguire, M. F. Goodchild, and D. W. Rhind, eds., Longman Group UK Ltd., Marlow, UK. 